\section{Francis Poisson} Philippe Roingeard Alain Goudeau

\section{Le virus de l'hépatite delta : un mode de réplication bien singulier}

Les caractéristiques du virus humain de l'hépatite delta (VHD) le distinguent de tous les virus du règne animal et l'apparentent aux petits $A R N$ pathogènes des plantes. L'une des particularités de ce virus est qu'il emprunte l'enveloppe du virus de l'hépatite $B$ (VHB) au cours de son cycle infectieux. Le génome du VHD code pour une unique protéine, l'antigène delta (AgHD), dont le rôle est essentiel dans la régulation du cycle de réplication. Deux formes de l'AgHD coexistent dans la cellule : l'AgHD $S$ a pour rôle d'amplifier la réplication du génome, alors que l'ÁgHD L l'inhibe et permet l'assemblage de la particule delta. L'AgHD interviendrait aussi dans l'interaction entre le VHD et le VHB et dans la pathogenèse de la co-infection. Ce virus étrange pourrait être un prototype de nouveaux agents infectieux, tant pour les animaux que pour les hommes.

\footnotetext{
ADRESSE

F. Poisson : docteur ès sciences. Ph. Roingeard : maître de conférences universitaire, praticien hospitalier. A. Goudeau : professeur des universites, praticien hospitalier. Département de microbiologie médicale et moléculaire Cnrs Ura 1334, unité de virologie, CHU Bretonneau, 37044 Tours Cedex, France.
}

e virus de l'hépatite delta (VHD) est un pseudo-virus à ARN tout à fait unique dans le monde animal. La découverte du VHD par le Dr Mario Rizzetto remonte à la fin des années 1970. Travaillant sur des biopsies hépatiques de malades atteints d'hépatite chronique $\mathrm{B}$, le Dr Rizzetto avait mis en évidence dans le noyau des hépatocytes un nouvel antigène. Des anticorps correspondant à cet antigène, dénommé antigène $\delta$, étaient détectables dans le sérum de malades souffrant d'hépatites chroniques B sévères [1]. Dans un premier temps, l'antigène $\delta$ avait été considéré comme un nouvel antigène du virus de l'hépatite $B$. Des expériences de transmission expéri- mentale au chimpanzé devaient montrer qu'il s'agissait d'une protéine codée par un agent pathogène distinct du virus de l'hépatite B (VHB), le virus de l'hépatite delta [2-4].

Dans les conditions naturelles de l'infection, le VHD ne se propage qu'en présence du virus de l'hépatite B dont il emprunte l'enveloppe. On observe ainsi des co-infections au cours desquelles les deux virus sont acquis simultanément et des surinfections par le VHD de malades déjà porteurs chroniques du virus B. Le VHD ressemble par bien des aspects aux viroïdes et aux petits $A R N$ pathogènes infectant les plantes $\left(\mathrm{m} / \mathrm{s} n^{\circ} 1\right.$, vol. 3, p. 55) $[5,6]$. Par ailleurs, ce pseudovirus empruntant l'enveloppe d'un virus auxiliaire se comporte un peu à 


\section{RÉFÉRENCES}

1. Rizzetto M, Canese MG, Arico J, Crivelli $O$, Bonino $F$, Trepo CG, Verme G. Immunofluorescence detection of a new antigenantibody system $(\delta /$ anti- $\delta$ ) associated to the hepatitis B virus in the liver and in the serum of HBsAg carriers. Gul 1977; 18:997. 1003.

2. Erlinger S. L'hépatite D. médecine/sriences $1985 ; 1: 64-5$

3. Bréchot C. L'agent delta : biologie et pathobiologie. médecine/sciences $1985 ; 1: 66-8$.

4. Bernuau J. Les hépatites dues au virus $D$ médecine/sriences $1985 ; 1: 69-73$.

5. Branch AD, Levine BJ, Roberston HD The brotherhood of circular RNA pathogens : viroids, circular satellites and the delta agent. Semin Virol $1990 ; 1$ : 143-52.

6. Diener TO. Autonomous and helper-dependent small pathogenic RNAs of plants viroids and satellites. In : Rizzetto M, Gerin JL, Purcell RH, eds. The hepatitis delta virus and its infertions. New York : Alan R Liss, $1987: 3-18$

7. Bonino F, Heermann $\mathrm{KH}$, Rizzetto $\mathrm{M}$ Gerlich WH. Hepatitis delta virus : protein composition of delta antigen and its hepatitis B virus-derived envelope. J Virol 1986 $58: 945-50$

8. Wang KS, Choo QL, Weiner AJ, Weiner AJ, Ou JH, Najarian RC, Thayer RM, Mullenbach GT, Denniston KJ, Gerin JL, Houghton M. Structure, sequence and expression of the hepatitis delta virus genome Vature $1986 ; 323: 508-14$. Correction : $N a-$ ture $1987 ; 328: 456$.

9. Kuo MYP, Chao M, Taylor J. Initiation of replication of the human hepatitis delta virus genome from cloned DNA : role of delta antigen. J Virol 1989; $63: 1945-50$.

10. Fu TB, Taylor J. The RNAs of hepatitis delta virus are copied by RNA polymerase II in nuclear homogenates. J Virol $1993 ; 67$ : 6965-72.

11. Ryu WS, Netter HJ, Bayer M, Taylor J. Ribonucleoprotein complexes of hepatitis delta virus. J Virol $1993 ; 67: 3281-7$.

12. Wu HN, Lai MMC. Reversible cleavage and ligation of hepatitis delta virus RNA Science 1989 ; 243 : 652-4.

13. Weiner AJ, Choo QL, Wang KS, Wang KS, Govindarajan S, Redeker AG, Gerin JL, Houghton M. A single antigenomic open reading frame of the hepatitis delta virus encodes the epitope (s) of both hepatitis delta

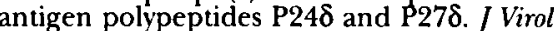
$1988 ; 62: 594-9$

14. Mac Naughton TB, Beard MR, Chao M, Gowans EJ, Lai MCC. Endogenous promoters can direct the transcription of hepatitis delta virus RNA from a recircularized cDNA

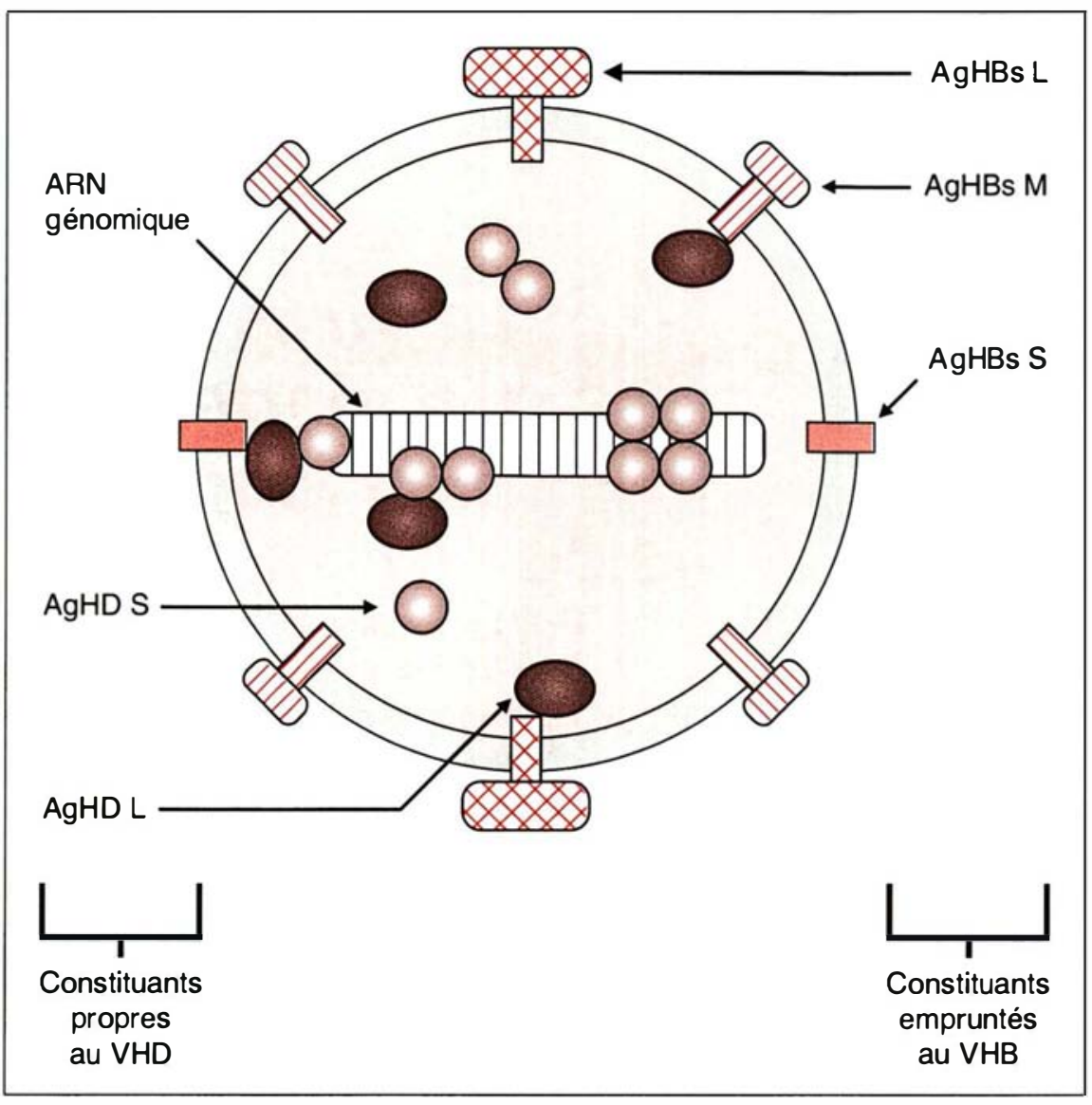

Figure 1. Structure de la particule virale $\delta$. AgHBs $S, M$ et $L$ : protéines d'enveloppe du virus de l'hépatite $B$. AgHD $S$ et $L$ : antigènes $\delta$.

l'image des virus satellites des virus à ARN des plantes [7]. Le génome du VHD, un ARN monocaténaire circulaire comprenant un fort degré d'appariement interne [8], ainsi que son mode de réplication en cercle roulant dans le noyau de la cellule infectée, confèrent au VHD les propriétés des viroïdes. Toutefois, à la différence des viroïdes dont le génome (250 à 400 nucléotides) est trop petit pour coder pour une protéine, le génome du VHD (1 700 nucléotides) code pour une protéine, l'antigène $\delta$ (AgHD) [9]. Enfin, le VHD s'apparente aux viroïdes par son autonomie de réplication. En effet, à l'opposé des ARN et virus satellites qui utilisent les enzymes de réplication de leurs virus auxiliaires, le VHD se réplique sans aucun facteur du VHB, dont le rôle se limite à fournir une enveloppe au génome du VHD [9 $10]$.

Le virus de l'hépatite delta appartient donc à une nouvelle classe d'agents inf ectieux, jusqu'alors insoupçonnée chez l'animal. Malgré cette remarquable économie de moyens, la réplication et la maturation du virus de l'hépatite delta sont finement réglées par l'antigène $\delta$ dont le rôle est essentiel dans le cycle viral. Au cours des cinq dernières années, la dynamique complexe de cette régulation et des interactions entre le VHD et le VHB a pu être en grande partie élucidée.

\section{La particule infectieuse}

Le virion complet infectieux, de $36 \mathrm{~nm}$ de diamètre, comprend l'ARN viral complexé à une vingtaine de 


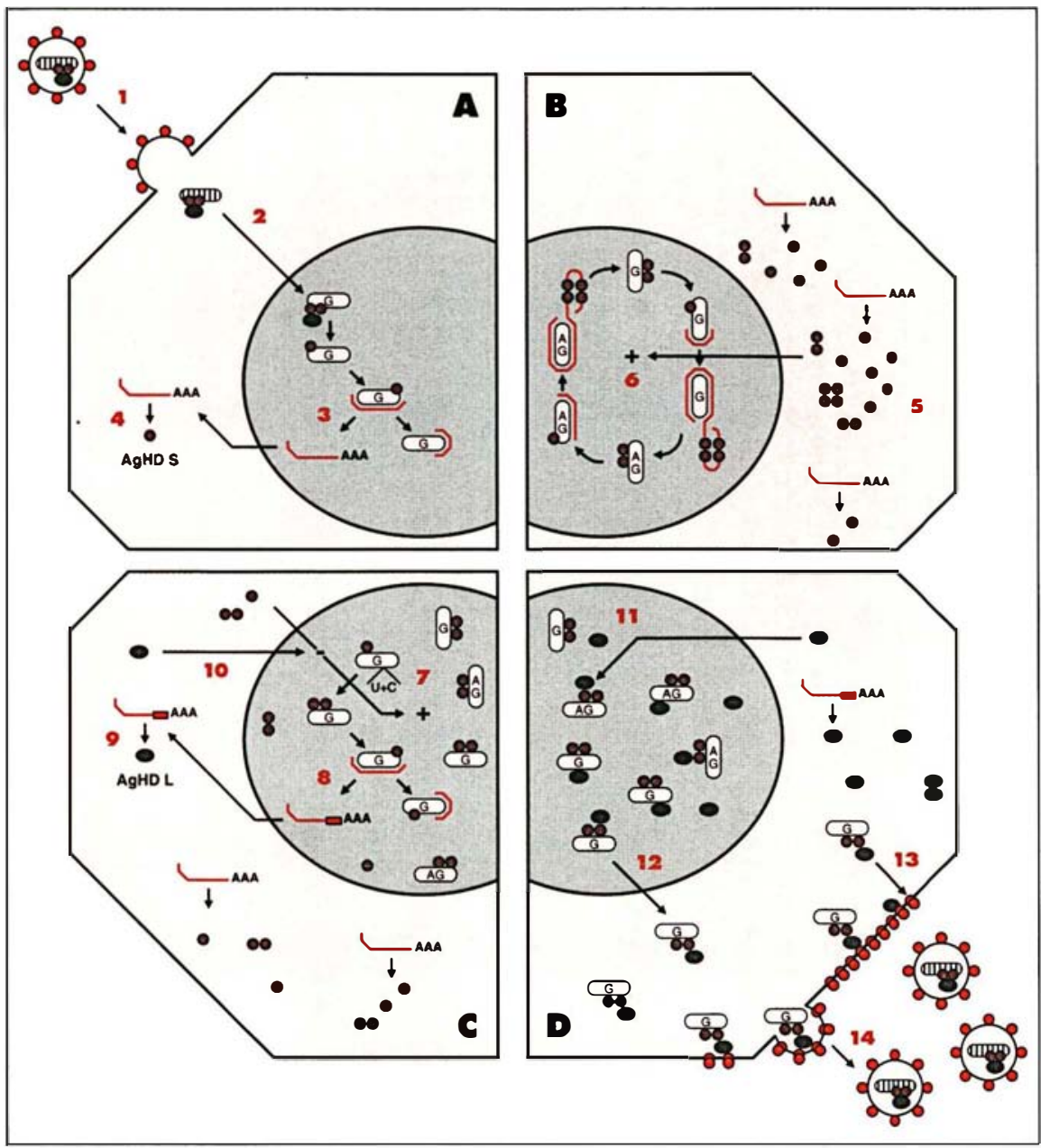

multimères d'AgHD et contient, en outre, quelques dizaines de molécules d'AgHD non liées à l'acide nucléique [11]. L'AgHD ne forme pas une capside structurée comme dans les virus conventionnels. Une molécule d'ARN et environ 70 molécules d'AgHD sont enveloppées dans une bicouche lipidique contenant l'antigène de surface (AgHBs) du VHB (figure 1).

L'ARN contenu dans les virions a une taille d'environ 1,7 kb. Il se présente sous la forme d'un brin monocaténaire circulaire fermé et possède environ $70 \%$ de bases appariées $(G+C)$ lui conférant une structure secondaire linéaire pseudo-double brin [8]. Un site spécifique d'autoclivage/autoligation, très important dans la réplication, est situé au nucléotide 688 (activité dite " ribozy$\mathrm{m} / \mathrm{s} n^{\circ} 10$, vol. 11 , octotre 95 me ") [12]. La molécule complémentaire de l'ARN génomique, $A R N$ antigénomique, est synthétisée dans la cellule infectée. Il possède également un site d'autoclivage/autoligation au nucléotide 901. Cinq à dix cadres de lecture ouverts codant pour plus de 100 acides aminés ont été identifiés dans le génome et l'antigénome du VHD. Seul l'AgHD codé par une séquence du brin antigénomique [8] est exprimé au cours de l'infection. L'AgHD est détecté, dans la particule infectieuse circulante et dans le noyau de la cellule infectée, sous deux formes de taille différente : une protéine de 195 acides aminés (forme $\mathrm{S}$ pour small) et une protéine de 214 acides aminés (forme L pour large) [7]. En effet, au cours de la réplication du VHD, une enzyme cellulaire encore non identifiée (une dé-
Figure 2. Cycle de multiplication cellulaire du VHD. A. Pénétration du virion et phase d'initiation de la réplication : (1) accrochage à la membrane de l'hépatocyte et pénétration du contenu du virion ; (2) transport nucléaire du brin génomique par l'AgHD ; (3) synthèse d'un ARNm antigénomique ; (4) traduction de I'ARNm dans le cytoplasme. B. Phase de réplication active : (5) synthèse de I'AgHD $S$ en grande quantité ; (6) activation de la réplication par I'AgHD S, synthèse de brins génomique et antigénomique. C. Phase d'inhibition de la réplication : (7) édition : mutation d'une base $U$ en $C$ sur le brin génomique ; (8) synthèse d'un ARNm antigénomique ; (9) synthèse de l'AgHD L ; (10) inhibition en trans de l'action activatrice de I'AgHD S. D. Assemblage des virions : (11) formation d'hétérodimères AgHD $S$ et $L ;(12)$ sélection du brin génomique ; (13) interaction entre l'AgHD L et l'AgHBs ; (14) formation des virions. roulase) induit une mutation dans un codon stop et l'apparition de génomes variants codant pour l'AgHD L correspondant à l'AgHD d'origine de S, prolongé en C-terminal de 19 acides aminés [13].

\section{Cycle de réplication du VHD}

La réplication débute par l'adhérence et la pénétration du virion dans l'hépatocyte (figure 2A). La ribonucléoprotéine est transportée du cytoplasme vers le noyau, par l'intermédiaire de l'AgHD qui comporte un signal de localisation nucléaire. $\mathrm{Ce}$ transport est très important car l'enzyme utilisée pour la réplication virale est localisée dans le noyau. L'ARN du VHD est répliqué par l'ARN polymérase II cellulaire [10] (qui norma- 


\section{RÉFÉRENCES}

15. Chen PJ, Kalpana G, Goldberg J, Goldberg J, Mason W, Werner B, Gerin J, Taylor $J$. Structure and replication of the genome of the hepatitis $\delta$ virus. Proc Nall Acad So USA $1986 ; 83: 8774-8$.

16. Hsieh SY, Taylor J. Regulation of polyadenylation of hepatitis delta virus antigenomic RNA. J Virol 1991 ; 65 : 6438-46.

17. Taylor J. Hepatitis delta virus : cis and trans functions required for replication. Cell $1990 ; 61: 371-3$

18. Lazinski DW, Taylor J. Relating structure to function in the hepatitis delta virus antigen. J Virol $1993 ; 67: 2672-80$.

19. Chang MF, Baker SC, Soe LH, Kamahora T, Keck JG, Makino S, Govindarajan S, Lai MMC. Human hepatitis delta antigen is a nuclear phosphoprotein with RNA binding activity. I Virol $1988 ; 62$ : 2403-10.

20. Chang MF, Chang SC, Chang CI, Wu K, Kang HY. Nuclear localisation signals, but not putative leucine zipper motifs, are essential for nuclear transport of hepatitis delta antigen. J Virol $1992 ; 66: 6019-27$.

21. Wang JG, Lemon SM. Hepatitis delta virus antigen forms dimers and multimeric complexes in vivo. J Virol $1993 ; 67: 446-54$.

22. Chen PJ, Chang FL, Wang CJ, Lin CJ, Wu JC, Sung SY, Chen DS. Functional study of hepatitis delta virus large antigen in packaging and replication inhibition : role of the amino-terminal leucine zipper. I Virol $1992 ; 66: 2853-9$.

23. Xia YP, Lai MMC. Oligomerization of hepatitis delta antigen is required for both the trans-activating and trans-dominant inhibitory activities of the delta antigen. J Viral $1992 ; 66: 6641-8$.

24. Chang MF, Chen CJ, Chang SC. Mutational analysis of delta antigen : effect on assembly and replication of hepatitis delta virus. J Virol $1994 ; 68: 646-53$.

25. Halazonetis TD, Georgopoulos $K$ Greenberg ME, Leder $P$. c-Jun dimerizes with itself and with c-Fos forming complexes of different DNA binding affinities. Cell $1988 ; 55: 917-24$.

26. Schuermann M, Neuberg M, Hunter JB, Jenuwein T, Ryseck RP, Bravo R, Muller R. The leucine repeat motif in Fos protein mediates complex formation with Jun/AP-1 and is required for transformation. Cell $1989 ; 56: 507-16$.

27. Lin JH, Chang MF, Baker SC, Govindarajan S, Lai MMC. Characterization of hepatitis delta antigen : specific binding to hepatitis delta virus RNA. J Virol 1990 ; 64: 4051-8.

28. Poisson F, Roingeard P, Baillou A, Dubois F, Bonelli F, Calogero RA, Goudeau A. Characterization of RNA-binding domains of hepatitis delta antigen. J Gen Virol 1993 ;

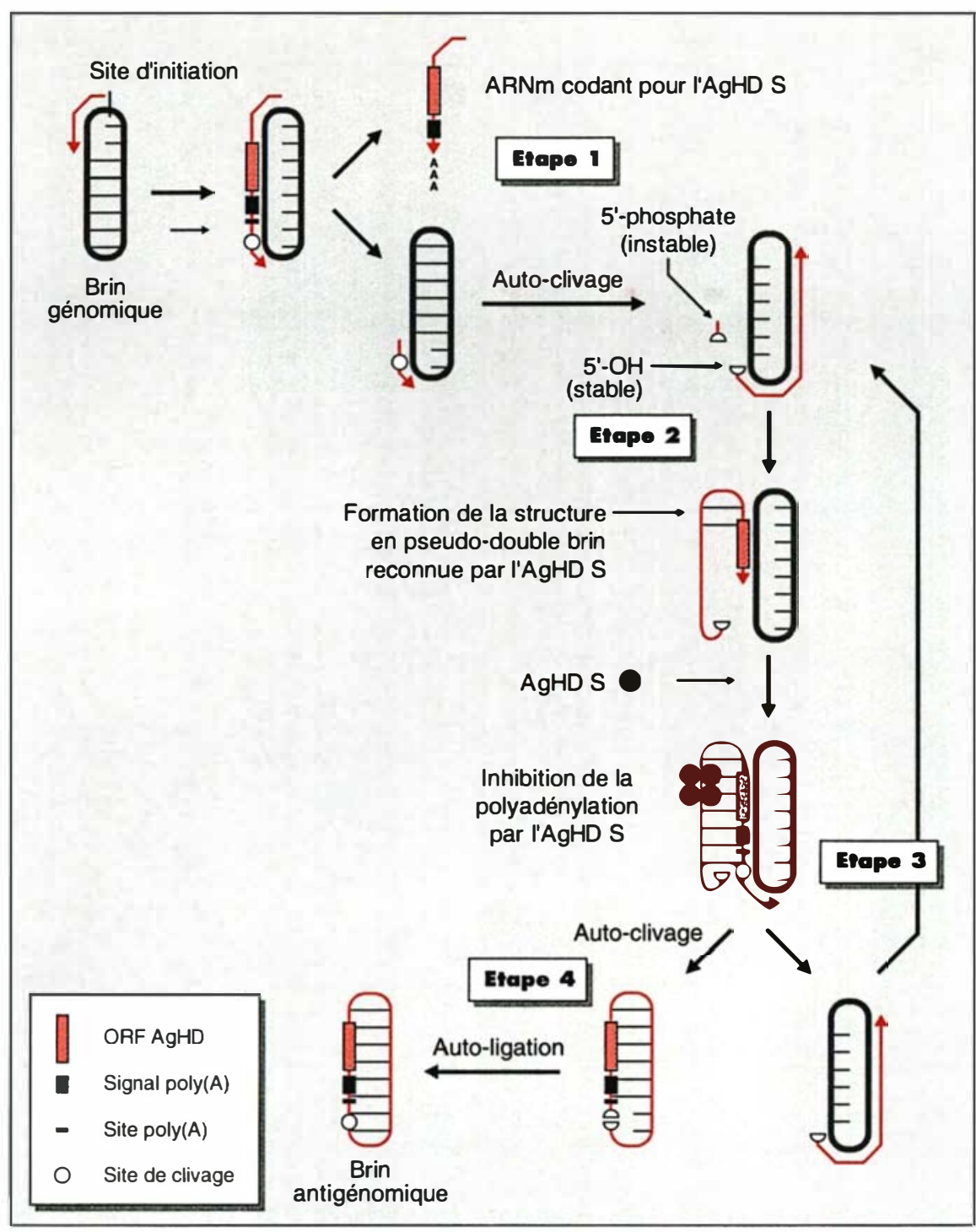

Figure 3. Modèle de régulation de la synthèse du brin antigénomique lors de la phase d'initiation de la réplication du génome du VHD. Le cycle de réplication du VHD comporte trois étapes : (1) synthèse d'un brin antigénomique. Un signal de polyadénylation permet d'engendrer un transcrit partiel mûri en ARNm qui sera traduit en AgHD S dans le cytoplasme; (2) la réplication continue en "cercle roulant " et le transcrit naissant subit un autoclivage produisant une extrémité $5^{\prime} \mathrm{OH}$ stable; (3) I'ARN engendré se structure en pseudodoubles-brins sur lesquels se fixent les molécules d'AgHD S, inhibant la poIyadénylation et permettant ainsi la synthèse d'un multimère de brins antigénomiques. Ce multimère est clivé en brins antigénomiques monocaténaires qui servent de matrice à la synthèse de brins génomiques au cours de la phase suivante. 


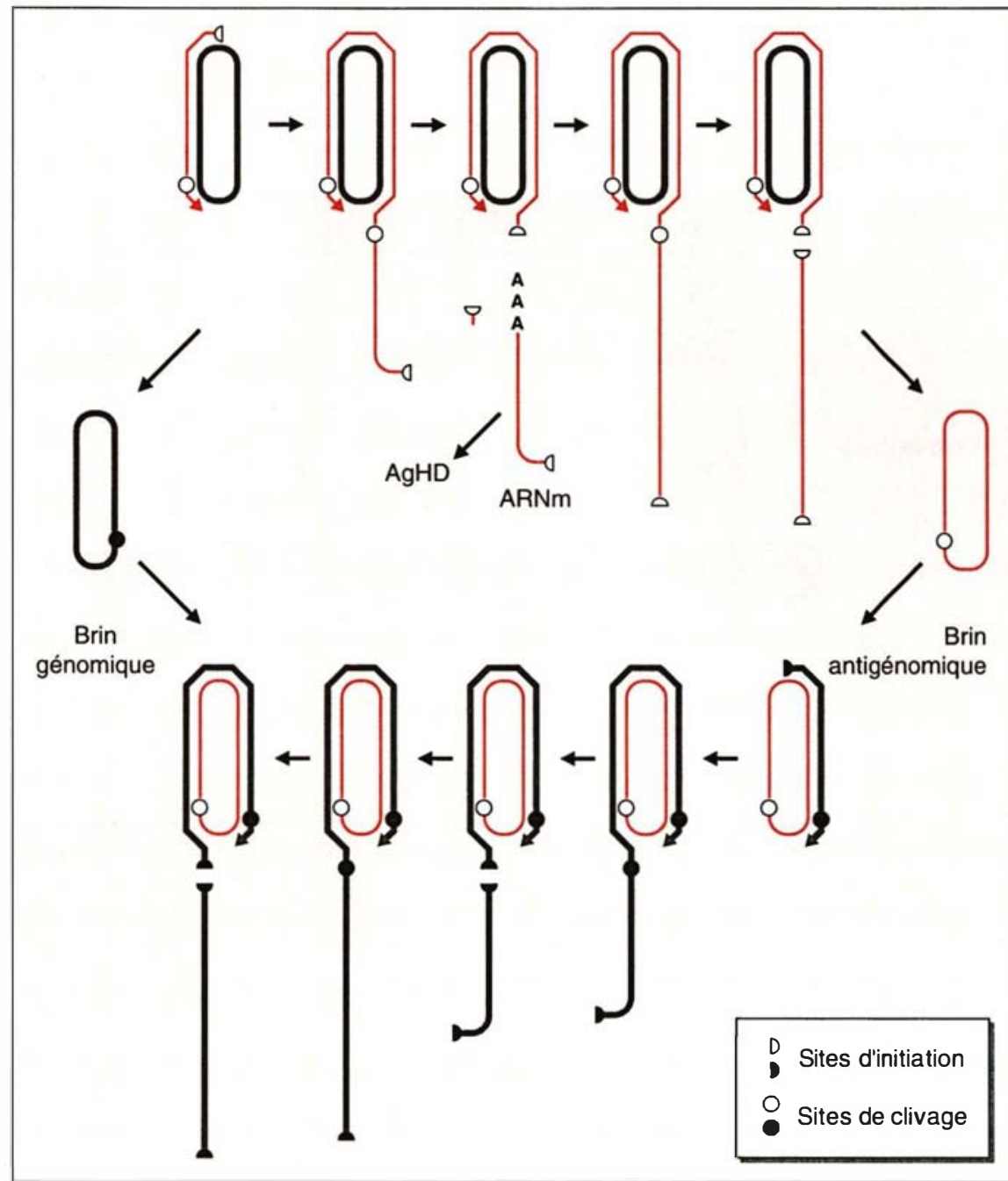

Figure 4. Mécanisme de réplication en u cercle roulant ". Les monomères génomiques et antigénomiques sont engendrés à partir des multimères de réplication grâce à des séquences spécifiques d'autoclivage et d'autoligation.

lement utilise un ADN double brin comme matrice), la structure d'ARN apparié permettant de tromper la machinerie cellulaire [14]. Dans l'hépatocyte infecté, l'ARN génomique et l'ARN antigénomique sont détectés sous forme linéaire et circulaire ainsi que sous forme de multimères (dimères et trimères) [15]. La présence de ces multimères s'explique par une réplication dite "en cercle roulant" [15].

Le cycle de réplication du VHD comporte trois étapes successives :

- Au cours de la première phase d'initiation (figure $2 A$ ), l'ARN polymérase reconnaît un promoteur sur le $\mathrm{m} / \mathrm{s} n^{\circ} 10$, vol. 11 , octobre 95
d'AgHD S. Cette liaison induit l'inhibition de la polyadénylation et permet ainsi la synthèse d'un multimère de brins antigénomiques (figure 3 : étape 3 ). Ce multimère est clivé en brins antigénomiques monocaténaires (figure 3 : étape 4) qui servent de matrice à la synthèse de brins génomiques au cours de la phase suivante [16].

- La phase de réplication active se déroule suivant le modèle du cercle roulant (figure 4). Les monomères génomiques et antigénomiques sont produits à partir des multimères de réplication grâce aux séquences spécifiques d'autoclivage/autoligation. A ce stade, le multimère d'AgHD S lié aux molécules d'ARN viral joue un rôle majeur d'amplification de la réplication par un mécanisme encore non identifié [17] (figure 2B). Ce mécanisme est extrêmement efficace puisqu'une cellule infectée peut contenir jusqu'à 300000 copies d'ARN génomique du VHD [15].

- Après cette phase d'amplification, une enzyme cellulaire (déroulase) induit une mutation sur l'ARN génomique du VHD, le codon stop UAG du cadre de lecture de l'AgHD S est transformé en codon tryptophane UGG. Cette mutation entraîne la synthèse d'ARN messager antigénomique codant non plus pour l'AgHD S (195 acides aminés) mais pour l'AgHD L (214 acides aminés). L'apparition de l'AgHD L entraîne l'inhibition en trans de l'action activatrice de la réplication de l'AgHD S (jigure $2 C$ ), là encore par un mécanisme non identifié.

brin génomique permettant la synthèse d'un brin antigénomique. En aval de la séquence codant pour l'AgHD un signal de polyadénylation conduit à engendrer un transcrit partiel mûri en un ARN poly(A) (ARN messager) qui est dirigé vers le cytoplasme pour être traduit en AgHD S (figure 3: étape 1). La réplication en cercle roulant continue, engendrant le site de clivage situé 50 nucléotides en aval du site de polyadénylation. Le transcrit naissant subit alors un autoclivage qui produit une extrémité 5'$\mathrm{OH}$ stable (figure 3 : étape 2). L'ARN naissant est capable de se structurer en pseudo-double brin, ce qui entraîne la fixation de molécule(s)
- L'apparition de l'AgHD L est l'élément déclenchant le processus d'assemblage du génome viral dans des particules néoformées (figure 2D). L'AgHD L, par la formation d'hétéromères avec l'AgHD S fixé sur l'ARN génomique, va permettre la connexion du complexe ribonucléoprotéique avec l'AgHBs situé au niveau du réticulum endoplasmique [18].

Ce mécanisme de réplication est extrêmement fin puisqu'il permet : dans un premier temps (AgHD S seul), d'amplifier la réplication virale dans la cellule infectée sans qu'il y ait excrétion de l'ARN viral, dans un deuxième temps $(\operatorname{AgHD} \mathrm{S}+\mathrm{L})$, de ra- 


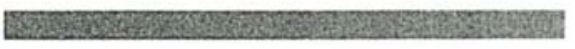

RÉFÉRENCES

29. Lee CZ, Lin JH, Chao M, McKnight $\mathrm{K}$, Lai MMC. RNA-binding activity of hepatitis delta antigen involves two arginine-rich motifs and is required for hepatitis delta virus RNA replication. J Virol $1993 ; 67: 2221-7$.

30. Poisson F, Roingeard P, Goudeau A. Mapping of the RNA binding domains of the hepatitis delta antigen. 1995 (soumis pour publication).

31. Glenn JS, Waston JA, Havel CM, White JM. Identification of a prenylation site in delta virus large antigen. Science 1992 ; 256 : 1331-3.

32. Hwang SB, Lai MMC. Isoprenylation masks a conformational epitope and enhances trans-dominant inhibitory function of the large hepatitis delta antigen. J Virol $1994 ; 68$ : 2958-64.

33. Zignedo AL, Dubois F, Samuel D, Georgeopolou U, Reynes M, Gentilini P, Bismuth A, Benhamou JP, Hadzyannis SJ, Bismuth H, Brechot C. Serum hepatitis delta virus RNA in patients with delta hepatitis and in liver graft recipients. $J$ Hepatol $1990 ; 11: 102-10$

34. Wu JC, Chen PJ, Kuo MYP, Lee SI Chen DS, Ting LP. Production of hepatitis delta virus and suppression of helper hepatitis $B$ virus in a human hepatoma cell line $J$ Virol 1991 ; 65 : 1099-104.

35. Negro F, Korba BE, Forzani IB, Baroudy BM, Brown TL, Gerin JL, Ponzetto A. Hepatitis delta virus (HDV) and woodchuck hepatitis virus (WHV) nucleic acids in tissues of HDV infected chronic WHV carrier woodchucks. J Virol 1989 ; 63 : 1612-8.

36. Rizzetto M, Ponzetto A, Forzani I. Epidemiology of hepatitis delta virus : overview. In : Gerin JL, Purcell RH, Rizzetto $M$, eds. The hepatitis delta virus. New York Willey-Liss Inc, 1991 : 1-20.

37. Dubois F, Goudeau A. Kinetics of delta antigen and antibody in acute delta hepatitis : evaluation with different enzyme im munoassays. J Clin Microbiol $1988 ; 26: 1339$ 42 .

38. Carreda F, Antinori S, Pastecchia C Coppin P, Palla M, Ponzetto A, Rizzetto M Moroni M. Incidence of hepatitis delta virus infection in acute HBs Ag negative hepatitis. Infect Dis $1989 ; 159: 977-9$.

39. Dubois F, Goudeau A, Roingeard P, Bacg Y, Guilmot JL, Choutet P. Diagnostic sérologique et épidémiologique des hépatites aiguēs delta en Indre-et Loire. Gastroenterol Clin Biol 1988; 12 : 887-93.

40. Buti M, Esteban M, Roggendorf M, Fernandez J, Jardi $R$, Rashofer $R$, Allende $H$ Genesca J, Esteban JI, Guardia J. Hepatitis D virus RNA in acute delta infection : serological profile and correlation with other markers of hepatitis D virus infection. Hepatolo

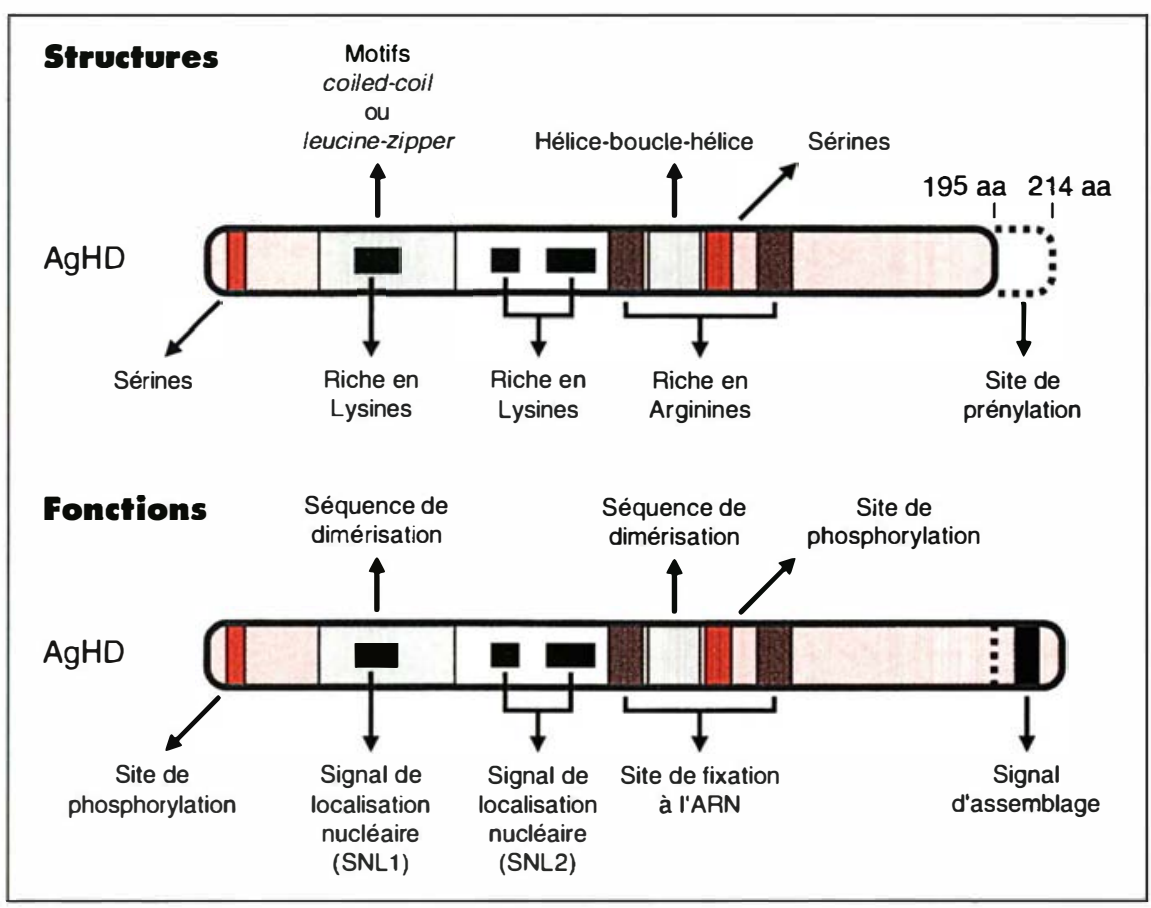

Figure 5. Structures et fonctions des AgHD S (195 acides aminés) et $L$ 1214 acides aminés). L'AgHD est phosphorylée sur des sérines appartenant à des sites consensus pour la caséine kinase II ; AgHD L est beaucoup plus phosphorylé que AgHD S. Les deux formes d'AgHD forment in vitro et in vivo des homo-et hétéromères par association de séquences type leucine zipper ou coiled-coil. La dimérisation des AgHD intervient dans l'activation (AgHD S) et l'inhibition (AgHD L) de la réplication, et l'empaquetage de AgHD S par $A g H D L$ pour former des particules. Les motifs riches en arginine permettent la liaison des AgHD à l'ARN du VHD en pseudo-doubles-brins. AgHD S active ainsi la réplication. AgHD $S$ et $A g H D L$ ne diffèrent que dans leur partie $C$ terminale qui comporte, pour AgHD L, un site de prénylation associé à la fonction de connexion du complexe ribonucléique à l'AgHBs.

lentir la réplication virale, probablement pour favoriser la survie de la cellule infectée, tout en permettant l'excrétion des virus néoformés dans la cellule infectée.

\section{Domaines fonctionnels de l'antigène $\delta$}

Les $\mathrm{AgHD} \mathrm{S}$ et $\mathrm{L}$ sont des protéines multifonctionnelles qui, du fait de leur séquence $\mathrm{C}$-terminale différente, jouent des rôles différents dans le cycle de réplication du VHD (figure 5).

\section{Sites de phosphorylation}

L'AgHD est une protéine phosphorylée au niveau de certains résidus sé- rines [19]. Deux sites " caséine-kinase II ", présents sur l'AgHD, possèdent des analogies avec la séquence consensus (S-X-X-D/E)* reconnue par la caséine-kinase II cellulaire : 2SRSE-5* et 123-SREE-126* [20]. La phosphorylation de l'AgHD pourrait intervenir dans le transport nucléaire, dans la régulation de la transcription, dans la sélection du brin génomique lors de l'encapsidation, dans l'interaction entre les AgHD et dans

\footnotetext{
* Code à une lettre des acides aminés : $A: A l a ; C$ Cys $; D: A s p ; E: G l u ; F: P h e ; G: G l y ; H: H i s$ $I: I l e ; K: L y s ; L:$ Leu ; $M: M e l ; N: A s n ; P$ Pro; $Q: G l n ; R: A r g ; S: S e r ; T: T h r ; V:$ Val $W: T r p ; Y: T y r$
} 
la capacité de fixer l'ARN du VHD. De plus, la différence de phosphorylation entre l'AgHD L et S (L six fois plus phosphorylé que $\mathrm{S}$ ) serait en corrélation avec leur fonctionnalité différente.

\section{Domaine de dimérisation}

Les deux formes d'AgHD forment in vivo et in vitro des homo- et hétéromères [21]. Cette fonction de dimérisation des AgHD est associée à une séquence $\mathrm{N}$-terminale de type leucinezipper ou coiled-coil suivant les isolats utilisés [21, 22]. Ces séquences sont constituées d'un agencement régulier d'acides aminés leucine ou isoleucine qui, organisés en hélice $\alpha$, permettent l'établissement d'interactions hydrophobes avec une structure homologue. Un autre domaine en position 108-122 possède une structure " hélice-boucle-hélice " considérée comme un élément permettant la dimérisation de certaines protéines [23].

La dimérisation des AgHD intervient dans (1) l'activation de la réplication de l'ARN du VHD par l'AgHD S qui agirait sous forme d'un complexe actif [23] ; (2) l'inhibition de la réplication par l'AgHD L, soit par la formation d'hétéromères AgHD S et $\mathrm{L}$ inactif, soit par compétition pour des facteurs de transcription cellulaires [23] ; (3) l'empaquetage de l'AgHD S par l'AgHD L puisque l'AgHD S ne possède pas la capacité de former des particules [24] et (4) la capacité des AgHD de fixer l'ARN du VHD. En effet, la formation de dimères facilite l'interaction protéines/acides nucléiques [25, 26].

\section{Séquences de localisation nucléaire (SLN)}

Deux séquences de localisation nucléaire ont été identifiées dans l'AgHD : SLN1 : 35-RKLKKKIKKL* 44 et SLN2 : 69-PPAKKLR*-75 et 85PRKRP*-89 [20]. Ces séquences permettent le transport nucléaire de la ribonucléoprotéine ainsi que celui de l'AgHD S, synthétisé dans le cytoplasme.

\section{Motifs riches en arginines (MRA)}

L'AgHD est une protéine ayant la capacité de se lier spécifiquement à
l'ARN du VHD en pseudo-double brin [19]. Les domaines 2-27 et 79163 responsables de cette fonction [27, 28] possèdent des motifs riches en arginine (MRA) couvrant les résidus 2-10, 97-107 et 136-146. La séquence 97-107 est indispensable à la fixation de l'ARN alors que la séquence 136-146 semble avoir une importance plus limitée $[29,30]$. Cette fonction de fixation à l'ARN est nécessaire à l'AgHD S pour activer la réplication [29].

\section{Site de prénylation}

L'AgHD L a pour rôle de connecter le complexe ribonucléoprotéique à l'AgHBs. Cette fonction a été associée à la partie (-terminale de l'AgHD L où une " boîte $\mathrm{CXXX}$ " $\left(\mathrm{CRPQ}^{*}\right.$ dans l'AgHD) est disponible [31]. Cette séquence est reconnue par une prényltransf érase cellulaire permettant la fixation d'un acide gras géranylgéranyl (C20) sur la cystéine 211. Ces éléments (addition de 19 acides aminés C-terminaux, prénylation) permettraient d'exposer une séquence interagissant avec l'AgHBs par changement de conformation de l'AgHD L [32].

\section{Relation avec le virus de l'hépatite $B$}

Le VHD a une réplication autonome, sans intervention d'aucun facteur du VHB. Mais en l'absence d'inf ection concomitante par le VHB, génératrice d'enveloppe, l'ARN du VHD ne peut pas être excrété de la cellule. Chez certains transplantés hépatiques initialement infectés par le VHB et le VHD, la réinfection du foie par le VHD peut se faire sans réinfection par le VHB [33]. Toutefois, seul un très faible nombre de cellules sont infectées par le VHD au cours de ce type d'infection, et c'est seulement après la réinfection par le VHB que la diffusion du VHD dans le foie peut se faire.

Les études séro-cliniques de malades infectés par le VHB et le VHD, ainsi que le modèle de la marmotte américaine infectée naturellement par un virus comparable au VHB (et susceptible de surinfection expérimentale par le VHD humain) ont montré que le VHD inhibe la réplication du VHB. Le mécanisme de cette inhibition est encore mal compris. Chez les plantes, l'inhibition de la réplication d'un virus par un ARN ou un virus satellite est un phénomène bien connu, intervenant par une compétition pour l'enzyme de réplication. Un tel mécanisme est à exclure dans le cas du VHD et du VHB, les deux virus utilisant des enzymes différentes pour leur réplication. Au moins deux grands mécanismes peuvent être retenus : une étude in vitro a montré un effet inhibitcur du seul AgHD sur la réplication du VHB [34] ; deux hypothèses (non démontrées) ont été proposées pour expliquer cette inhibition: (1) l'AgHD pourrait se fixer sur les ARN du VHB et ainsi affecter leur stabilité ou empêcher la formation des virions ; (2) l'AgHD pourrait se lier, de façon directe ou indirecte par l'intermédiaire de facteurs de transcription, sur les séquences régulatrices de l'ADN du V'HB, entraînant une diminution de la transcription. Par ailleurs, le modèle marmotte a montré que le VHD inhibe la réplication du VHB de la marmotte se répliquant au niveau de la rate, alors qu'elle n'est pas infectée par le VHD [35]. Cet effet indirect pourrait être dû à la production d'interféron par la cellule infectée par le VHD, la présence d'ARN double-brin étant un facteur stimulant la synthèse d'interféron.

\section{L'hépatite delta}

\section{Épidémiologie}

Du fait que le VHD emprunte son enveloppe au VHB, l'infection par le VHD est dépendante de l'infection par le VHB. C'est pourquoi l'épidémiologie et les modes de transmission du VHD ressemblent en partie à ceux du VHB $[4,36]$. En Europe du Nord et de l'Ouest, aux États-Unis, où le portage chronique du VHB est peu fréquent $(<1 \%)$, l'infection par le VHD est essentiellement confinéc aux toxicomanes utilisateurs de drogues par voie parentérale.

\section{Co-infection}

Cliniquement, rien ne permet de distinguer une hépatite aiguë par co-infection VHB/VHD d'une hépatite aiguë par le VHB seul. Le diagnostic sérologique de la co-infection repose sur la présence d'Ag $\mathrm{HBs}$ et d'IgM 


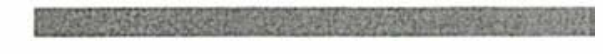

\section{RÉFÉRENCES}

41. Roingeard P, Dubois F, Marcellin P, Bernuau J, Bonduelle S, Benhamou JP, Goudeau A. Persistent delta antigenaemia in chronic delta hepatitis and its relation with human immunodeficiency virus infection. $J$ Med Virol $1992 ; 38$ : 191-4.

42. Buti M, Esteban R, Jardi R, Allende H, Guardia J, Roggendorf $M$. Chronic delta infection in a patient without detectable $\mathrm{HBs}$ $\mathrm{Ag}$ in serum. Hepatology $1988 ; 8: 985-6$.

43. Bonino F, Negro F, Baldi M, Brunetto MR, Chiaberge E, Capalbo M, Maran E, Lauarini C, Rocca N, Rocca G. The natural history of chronic delta hepatitis. In : Rizzetto M, Gerin JL, Purcell RH, eds. The he patitis delta virus and its infection. New York : Alan R. Liss Inc, 1987 : 145-52.

44. Buti M, Esteban R, Espanol MT, Malagelada A, Jardi R, Rodriguez Frias F, Roget M, Guardia M. Influence of human immunodeficiency virus infection on cell-mediated immunity in chronic D hepatitis. J Infect Dis $1991 ; 163: 1351-3$

45. Negro F, Gerin JL, Purcell RH, Miller R Sequence homologie between HDV RNA and 7SL RNA : implications for pathogenesis. In : Gerin JL, Purcell RH, Rizzetto M, eds. The hepatitis delta virus. New York : Willey Liss Inc, 1991 ; $364:$ 327-32.

46. Chao YC, Chang MF, Gust I, Lai M MC. Sequence conservation and divergence of hepatitis $\delta$ virus RNA. Virology $1990 ; 178$ : 384-92.

47. Chao YC, Lee CM, Tang HS, Govindarajan S, Laim MC. Molecular cloning and characterization of hepatitis delta virus from Taiwan. Hepatology 1991 ; 13 : 345-52.

48. Rosina F, Pintus C, Meschievitz C, Rizzetto $M$. A randomized controlled trial of a 12-months course of recombinant human interferon $\alpha$ in chronic delta (type D) hepatitis : a multicenter study. Hepatology 1991 ; 13 : 1052-6.

49. Farci P, Mandas A, Coina A, Lai ME, Desmet V, Van Eyken P, Gibo Y, Caruso L, Scaccabarozzi S, Criscuolo D, Ryff JC, Balestrier A. Treatment of chronic hepatitis D with interferon $\alpha$-2a. N Engl J Med 1994 ; 330 : 88 94.

50. Samuel D, Bismuth $H$, Benhamou JP. Liver transplantation in cirrhosis due to hepatitis D virus infection. J Hepatol 1993 ; 17 : S154-6.

51. Karyanis $P$, Saldanha J, Monjardino J Goldin R, Main J, Luther S, Easton M, Ponzetto A, Thomas HC. Immunisation of woodchucks with recombinant hepatitis delta antigen does not protect against hepatitis delta virus infection. Hepatology 1990

\begin{tabular}{|c|c|c|c|c|}
\hline \multicolumn{5}{|c|}{$\begin{array}{c}\text { TABLEAU I } \\
\text { MARQUEURS SÉRIQUES DES HÉPATITES DELTA }\end{array}$} \\
\hline & Co-infection & \multicolumn{3}{|c|}{ Surinfection } \\
\hline $\begin{array}{l}\text { Phase initiale } \\
\text { AgHBs } \\
\text { IgM anti-HBc } \\
\text { AgHD } \\
\text { Anti-HD } \\
\text { IgM anti-HD }\end{array}$ & $\begin{array}{c}\text { Positif } \\
\text { Positif } \\
\text { Transitoire } \\
\text { Séroconversion } \\
\text { Séroconversion }\end{array}$ & \multicolumn{3}{|c|}{$\begin{array}{c}\text { Positif } \\
\text { Négatif } \\
\text { Transitoire } \\
\text { Séroconversion } \\
\text { Séroconversion }\end{array}$} \\
\hline $\begin{array}{l}\text { Évolution } \\
\text { (>6 mois) } \\
\text { AgHBs }\end{array}$ & Négatif & \multicolumn{3}{|c|}{ Positif } \\
\hline $\begin{array}{l}\text { AgHD } \\
\text { Anti-HD } \\
\text { IgM anti-HD }\end{array}$ & $\begin{array}{l}\text { Cas général } \\
- \\
+ \\
-\end{array}$ & $\begin{array}{l}\text { Fréquent } \\
\qquad \begin{array}{l}- \\
+ \\
+\end{array}\end{array}$ & $\begin{array}{l}\text { Rare } \\
- \\
+ \\
-\end{array}$ & $\begin{array}{c}\text { Cas particulier* } \\
+ \\
\pm \\
\pm\end{array}$ \\
\hline
\end{tabular}

* Fréquent chez les sujets VIH-positifs.

anti-HBc de titre élevé (témoin d'une infection récente par le VHB) et des marqueurs du VHD : AgHD pendant une à trois semaines après le début de l'ictère puis anticorps anti-HD (Tableau I) ; tous les deux détectés par méthode immuno-enzymatique, $\mathrm{ARN}$ du VHD détecté par hybridation moléculaire [37]. Toutefois, du fait de l'inhibition de la réplication du VHB induite par le VHD, l'Ag HBs peut être indétectable dans le sérum pendant quelques semaines [38]. La coinfection VHB/VHD se caractérise par une tendance à une sévérité exacerbée de l'hépatite aiguë comparée à celle occasionnée par le VHB seul [39].

\section{Surinfection}

L'infection par le VHD chez un sujet chroniquement infecté par le VHB provoque une hépatite aiguë, quelquefois résolutive, mais l'éventualité la plus fréquente est le développement d'une infection chronique delta [4]. Si l'infection chronique par le VHB est connue, le diagnostic repose sur la mise en évidence d'une séroconversion pour les marqueurs du
VHD [39-41]. Sans la connaissance d'une sérologie VHB antérieure, le diagnostic repose sur la mise en évidence des marqueurs du VHD : AgHD à la phase précoce de l'infection VHD puis anticorps anti-HD ( $T a-$ bleau I) et de l'Ag HBs, en l'absence d'IgM anti-HBc (témoignant d'un contact ancien avec le VHB). Toutefois, le VHD inhibant la réplication du VHB, l'Ag HBs peut être indétectable pendant la phase aiguë de la surinfection, et parfois même pendant plusieurs mois [42]. L'établissement d'une infection chronique delta, marquée par la persistance des marqueurs du VHD, est caractérisé par une accélération très rapide de l'hépatite chronique vers une insuffisance hépatocellulaire (en un à trois ans) dans $15 \%$ à $20 \%$ des cas [43]. Il est probable que le VHD exerce le maximum de son effet pathogène dans les premières années de la surinfection ; pour les malades qui survivent à cette période, il semble que l'effet de la surinfection VHD sur l'évolution de l'hépatite chronique Ag HBs positive soit moins marqué. La surinfection par le VHD ne constitue pas, en elle-même, un risque 
accru de développement d'un carcinome hépatocellulaire.

\section{Pathogenèse de l'infection par le VHD}

Les mécanismes impliqués dans la pathogenèse du VHD sont encore mal connus. Des études histologiques ont suggéré un effet cytopathique direct : d'autres ont suggéré un mécanisme indirect, via une réponse immunitaire cellulaire $\mathrm{T}$, comme pour le $\mathrm{VHB}$. L'étude des perturbations hépatiques de malades atteints d'hépatite chronique delta avec ou sans immunodéficience acquise n'a pas montré de différences significatives [44]. Il est donc probable que l'immunité cellulaire $\mathrm{T}$ joue un rôle modeste dans l'agression hépatique observée au cours de l'hépatite delta.

Un effet cytopathique direct du V'HD pourrait s'exercer par plusieurs mécanismes : (1) une crtotoxicité directe de l'AgHD exprimé dans la cellule infectée ; ce mécanisme a été montré in vitro et semble spécifique de la forme S de l'AgHD; (2) une compétition entre l'ADN cellulaire et l'ARN du VHD pour l'ARN polýmérase 11: (3) l'existence d'une complémentarité entre l'ARN antigénomique du VHD et un ARN cellulaire, l'ARN 7SI impliqué dans la localisation des protéines membranaires, pouvant potentiellement conduire à la formation d'un hybride et à l'altération des fonctions cellulaires [45]. Ces deux dernières hypothèses ont déjà été proposées pour expliquer la pathogénicité des viroïdes chez les plantes, laquelle ne peut être la conséquence que d'un effet direct de l'accumulation d'ARN viral dans la cellule, les viroïdes ne codant pas pour des protéines et les plantes ne développant pas de réponse immune.

Toutefois, il est possible que ces trois types de mécanismes directs, ainsi qu'une réponse cellulaire $\mathrm{T}$ se superposent pour induire un effet cytopathogène. Egalement, la proportion entre génomes codant pour la forme S de l'AgHD et génomes codant pour la forme $\mathrm{L}$ pourrait intervenir dans le degré de cette pathogénicité.

Enfin, il faut noter que le séquençage de quatre isolats de VHD (États-Unis, Italie, Taiwan, îles du Pacifique) a montré des variations importantes
(11\% à $17 \%$ de variabilité) [46, 47]. Trois régions à caractère fonctionnel sont fortement conservées : (1) la séquence responsable de l'activité autocatalytique de l'ARN génomique ; (2) celle responsable de l'activité autocatalytique de l'ARN antigénomique ; (3) la région de l'antigénome codant pour la partie de l'AgHD responsable d'interactions avec l'ARN du VHD [47]. La variabilité observée sur le reste du génome et de l'antigénome pourrait être impliquée dans des différences de pathogénicité des isolats, expliquant la tolérance de certaines populations pour le VHD, comme cela semble être le cas sur le pourtour méditerranéen et dans les îles du Pacifique. Des variations pourraient également se produire dans le temps, au cours de l'infection chronique chez un sujet donné, et conduire à une atténuation de la pathogénicitcé.

\section{Traitement et prévention de l'infection par le VHD}

Plusicurs études ont montré l'efficacité partielle d'un traitement par l'interféron $\alpha$, la diminution de la virémic et la normalisation des transaminases sériques che\% $30 \%$ à $70 \%$ des malades traités étant le plus souvent des événements réversibles à l'arrêt du traitement [48, 49]. La transplantation hépatique donne des résultats satisfaisants ; probablement du fait de l'inhibition de la réplication du V'HB par le VHD, la réinfection du foie par le VHB après transplantation intervient moins souvent que dans le cas d'une infection par le VHB seul [50].

Du point de vue de la prévention, elle est identique à celle du VHB. La vaccination contre le VHB, qui neutralise les interactions entre l'enveloppe du VHB (et donc du VHD) et l'hépatocyte, protège de la co-infection IHB/VHD. Pour un sujet porteur chronique du VHB, la protection contre la surinfection delta ne peut reposer que sur des mesures d'hygiène, qui sont fonction de l'appartenance à un groupe à risque. La mise au point d'une vaccination spécifique contre le VHD n'est pas envisageable, le modèle de la marmotte ayant montré que les anticorps anti- $\delta$ ne protègent pas de l'infection par le VHD [51].

\section{Conclusion}

La découverte du VHD a permis de mettre en évidence au sein du monde animal l'existence d'agents infectieux s'apparentant aux viroïdes et aux autres petits ARN pathogènes du monde végétal. La seule protéine codée par ce virus joue un rôle complexe dans le mécanisme de réplication de l'ARN viral. Complètement autonome pour sa réplication dans la cellule infectée, le VHD ne peut mener un cycle infectieux complet qu'avec l'aide du virus de l'hépatite B : l'ARN du VHD est alors excrété de la cellule infectée en s'entourant de l'enveloppe du VHB. La mise en évidence d'un tel virus représente peutêtre une porte ouverte vers l'identification d'agents infectieux similaires en pathologie humaine

\section{Surnmary}

The hepatitis delta virus: a singular réplication

The human hepatitis delta virus (HDV) is an unusual infectious agent of animals showing many similarities to pathogenic RNAs of plants. Some unique and intriguing features have been assigned to HDV : the envelope of an helper virus, the hepatitis $\mathrm{B}$ virus (HBV), is needed for the assembly of HDV particles ; its genome encodes a solitary protein, the delta antigen, which is essential to the regulation of the viral genome replication and the viral genome packaging into HBV envelope. In fact two forms of the delta antigen appear sequentially in the infected cells : the small form, that positively regulates HDV replication, and the large form, that negatively regulate HDV replication but promotes the particle assembly into $\mathrm{HBV}$ envelope. The possibility remains that HDV could be a prototype for a class of yet unknown infectious agents, that might be involved in other diseases of animals.

\section{TIRÉS À PART}

F. Poisson 